\title{
How will IoT Influence the Quality of Life for Silver Population?
}

\author{
Mihaela Cazacu \\ Alexandru Hanță \\ Emilia Ţiţan \\ The Bucharest University of Economic Studies \\ Romania
}

\begin{abstract}
IoT is a concept that has emerged in recent years bringing significant changes in technology, social and economic sectors. Daily activities of the population are thus enhanced by the Internet connection and the power of data analysis, elements that will change the way we live, work or act in the future. The projections made by IoT experts especially from economic point of view are impressive. Until 2025, revenues of up to $\$ 11$ trillion and more than 100 billiards of connected devices are expected.

For silver population, the impact of the Internet on all Things will be strong especially in Smart Health technologies that will have the role of preventing and treating effectively existing diseases. The economic impact of smart solutions in the health sector has reached European level at about 10\% of the Gross Domestic Product, but in the coming years there is a significant increase in revenue in this sector ${ }^{l}$.

Also, from the quality of life point of view, smart technologies will significantly improve human activity, but it can be a zero-sum game, and pollution and environmental impact can have harmful effects whose impact cannot yet be measured.
\end{abstract}

Keywords: Internet of Things (IoT), quality of life, silver population, economic impact

\section{Introduction}

For sure the appeareance of the Internet has had a strong global impact on distance and time with other dimensions through the power of the connection, but in the future there is a big transformation in its use, with users maturing enough to discover smart technologies that ease their daily activity. The most exciting predictions about the future of the Internet come from big companies that are trying to diversify their service portfolios, so Ericson claims that by the end of 2020 more than 50 billion devices will be connected while Cisco predicts growth of the Internet as a dimension of about 20 times (The Internet of Things, Björn Raunio, 2009).

The aim of this paper is to show the impact of the Internet of all Things on the quality of life of the silver population. In 2015, the European Commission launched a reference document on the silver economy The silver economy is presented with four broad areas of application and application of the concept: first of all, the prospecting of the labor market for the elderly, the adaptation of the environment and the means of transport , increased lifespan, health monitoring methods (The silver economy, Opportunities from aeging, July 2015).

Cisco's Visual Networking Index study shows that internet of things will grow faster than another category of interconnected devices. At the global level, device-to-device connections will increase approximately threefold, from 4.9 billion in 2015 to 12.2 billion in 2020 . Also, the medical area will increase up to five times, making it the fastest 144 million connections in 2015 to 729 million in 2020 (Cisco Visual Networking Index TM (VNI) Complete Forecast for 2015 to 2020, 2016). The Internet of Things refers to the representation of all objects in an internet network. The term was first introduced in 1999 by Ashton (That's "Internet of Things" by Thing, K. Ashton, RFID Journal, 2009), but there is no unanimously accepted definition, the term Internet of Things being often associated with progress from technologic point of view. 
The „Things” refer to virtual objects that offer one or more services, basically reflecting the shift from production and consumption of data from the internet from people to interconnected machines meant to make a considerable improvement in people's daily activity, thus enabling the first rule of thumb of the future, according to which "anything can connect, it will connect" (A Simple Explanation of 'The Internet Of Things', Forbes). Thus, daily activity becomes smart, anticipating and relieving it. One of the best examples in this respect is "smart city", the city of the future where traffic is monitored and fluidized with the help of the internet, as well as reduced energy consumption.

The Internet of Things comes with a number of opportunities, but many more challenges. If excessive technology consumption will generate the flourishing of various machine-building sectors, challenges will arise from the point of view of the security of the shared information, the architecture of data sharing systems that will support a huge amount of data and the management and storage of such data, the environment as production increases.Basically, how does this concept work? Integration of each device with the internet will make the unique IP address of the device a unique identifier. But the Internet of all things will not just mean embedded devices and sensors, it will also include devices for wider operations, such as routers, data processing systems.

In 2015, the global population had reached 7.3 billion, while in 2050 an increase of up to 10 billion (the French Institute for Demographic Studies) is expected, of which about $17 \%$ of the elderly over 65 compared to around $8.5 \%$ today (The US Bureau of Statistics), which is why there is real interest in the medical and social system, the labor market or the transport system and their adaptation to the needs of the elderly.

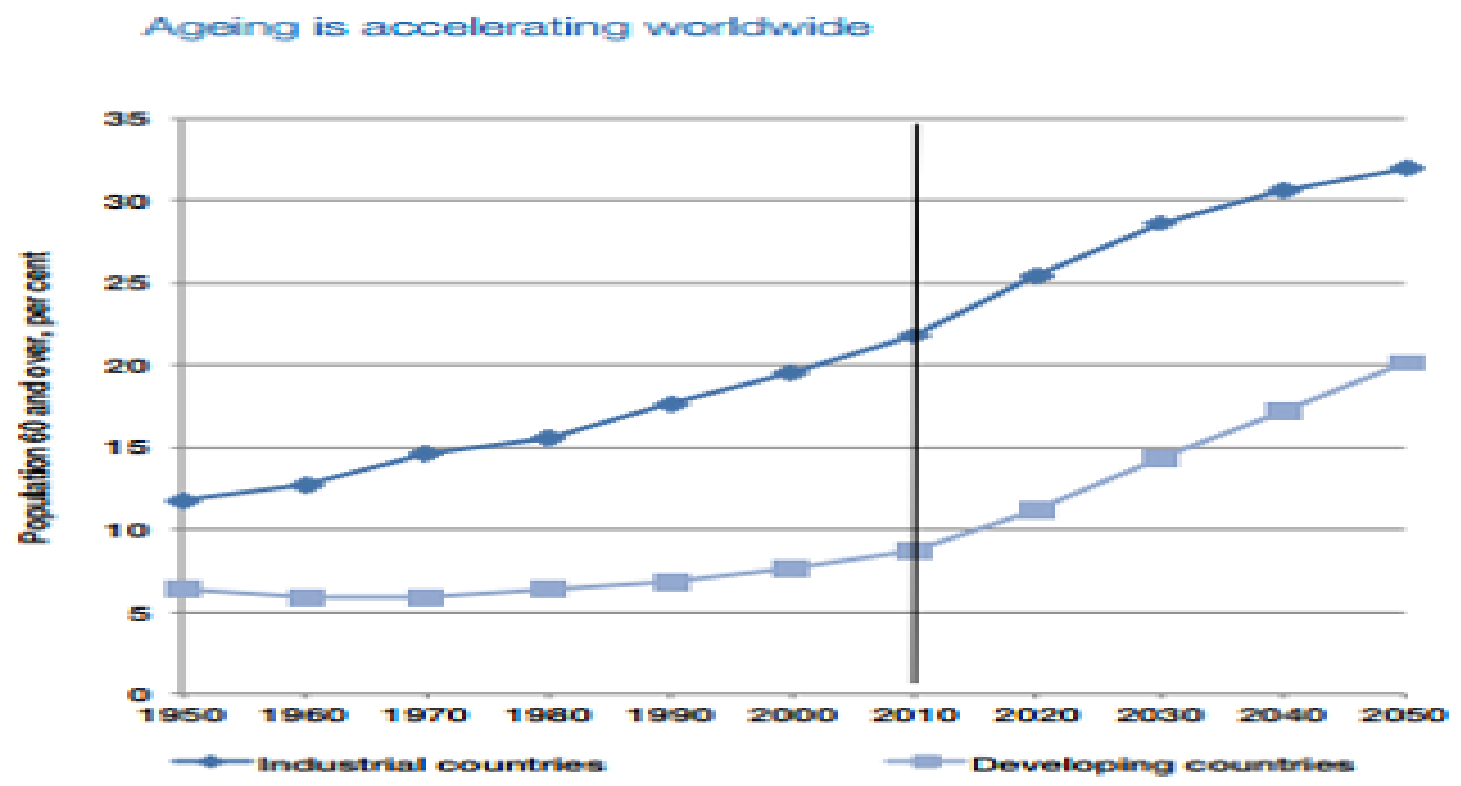

Source: UN, World Population Prospects: The 2010 Revision (http://www.un.org/en/index.html)

The problem of the growth of the elderly population concerns both developed and developing countries, thus demysizing the fact that the phenomenon of aging affects only developed countries (The seven myths of population aeging, Accenture, 2012).

Silver population's quality of life is strongly influenced by technological developments planned for the future, quality of life is basically individual perceptions of their social, within the context of cultural values in living and depending on their needs, standards and aspirations, according to a definition of World Health Organization in 1998. One of the immediate applications for the aging population can refer to the tools used to monitor, diagnose and offer online health support, thus bringing a paradigm shift into the medical system. This is not the only application of the internet of things for this population, transportation system and labor market adjustment may be other examples suitable for introduction of intelligent systems and facilitate growth of elderly population satisfaction on the quality of their life. Also, by using the Internet to a large extent, it is demystified that the elderly population is more adaptable with the use of modern technology (The seven myths of population aeging, Accenture, 2012). 
At the EU and candidate countries level, considering the percentage of individuals using different tools to compete on the internet, there is over $60 \%$ use in Norway, one of the most highly-developed countries at European level.

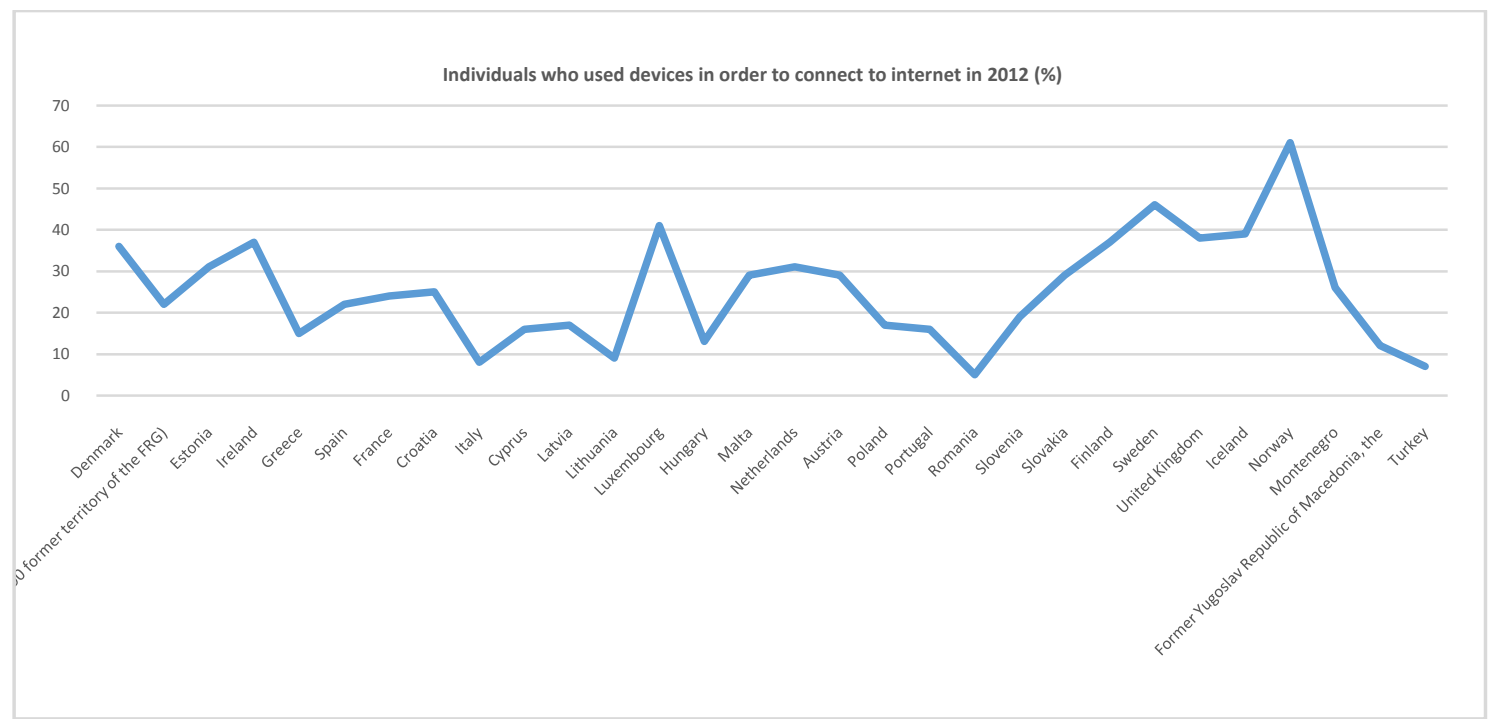

Source: Eurostat (http://ec.europa.eu/eurostat)

Also, considering the reasons for accessing the Internet for seeking health information, there has been an increasing trend over the last 10 years, once again emphasizing the effect of IoT and its applicability in everyday life.

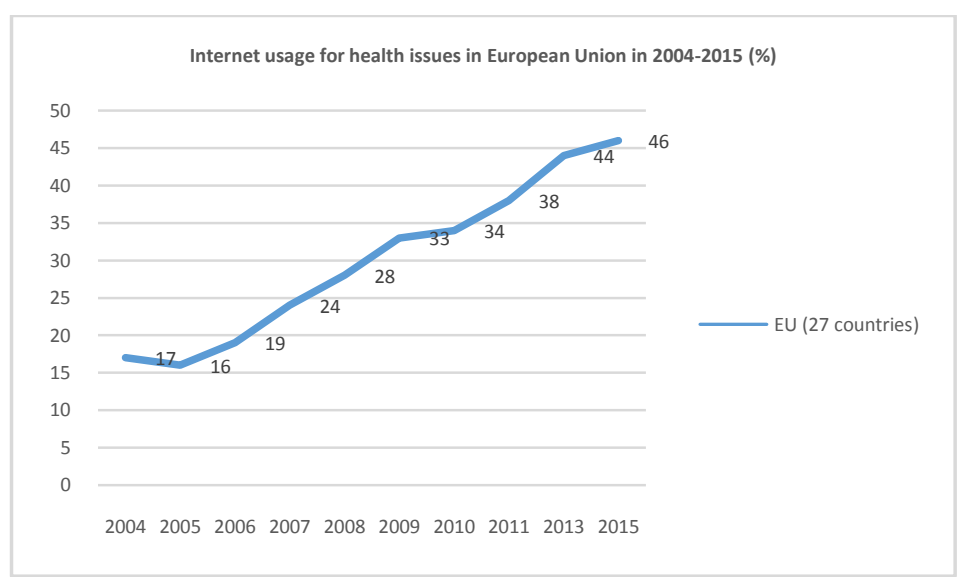

Source: Eurostat (http://ec.europa.eu/eurostat)

The phenomenon named „Internet of all Things” is in full swing and implementation. Impact is visible in increasing the quality of life, especially for the elderly by facilitating access to daily activities.Below, we will present a case study on the concept of "smart city". To be smart, a city has to meet several features in three important areas: administrative, technological and social. The administrative aspect aims at decongestion of traffic, while the technological aspect, the investment in technology. The social aspect is a community one and it means maximizing synergy between citizens.

The concept of smart city solves a wide range of problems facing today's cities, mentioning issues such as urban stress generated by population growth, inappropriate infrastructure, but the most important economic competition is related to environmental issues.

At European Union level, the European Economic and Social Committee (EESC), an EU consultative body composed of representatives of workers 'and employers' organizations and other interest groups, was set up and one of the main tasks was to ensure that EU policies and legislation were adapted economic and social conditions, seeking a consensus that serves the common good. 
The six pillars on which the European Economic and Social Committee is based are:

- Technologies and tools to improve energy efficiency and integrate renewable energy sources

- Large-scale development of information and communication technologies

- Technology and connectivity platforms for the creation of new digital services systems to improve the quality of life and work of citizens

- Infrastructure adaptation and urban redesign

- Sustainable Sustainable Investment Models

Among the areas of a smart city are: electricity being the essence of moving things, telecommunication, transport, water and domestic water, public safety. The relationship between the elderly and intelligent cities is under the sign of several dimensions including housing, transport, social participation, social inclusion, healthcare, communication systems, leisure and culture so that the environment becomes more elderly friendly and they become more more comfortable with it. For example, at the European level, the WeCare program has been created to develop online social services to help communicate and connect elderly people with friends and family members, neighbors, Internet use by the elderly to better integrate them into society.

To support the elderly and their needs at European level, the City4Age project was created as part of Horizon 2020, designed to support the interests of the elderly. The program is coordinated by the Polytechnic University of Milan and originally comprised a pilot project tested in six cities in different geographical areas: Madrid, Athens, Montpellier, Singapore, Lecce and Birmingham. In conclusion, the internet concept of all things is beneficial for the elderly, helping to integrate into society, familiarize with technology and at the same time facilitate agespecific activities.

Also, if we are looking for the economic impact of Internet of Things, at the World Economic Forum Annual Meeting in Davos this year, were presented two major results of some researches having the aim to present the impact of IoT for society and UN's sustainable development goals, goals which should cover UN's mission for 2030:

- For sustainability the Internet of Things could be a game changer

In this case the attention is focused on good health and well-being (7\%), smart cities and communities (19\%).

- Measurement of the actual projects is really challenging

Based on the research results, $95 \%$ of the IoT deployments are small or medium scale.

Based on reports presented in Davos, artificial intelligence and internet of things are two important topics nowdays and for sure the focus will be on the measurement and also the impact generated for society. Smarter cities, cleaner air and water, smart agriculture, cutting food waste, fighting cancer or connecting patients are already part of our lives and those are some simple examples of how Internet of Things and artificial intelligence will improve lives around the world.

\section{References}

The Internet Society (ISOC), The Internet of Things: An Overview. Understanding the Issues and Challenges of a More Connected World, October 2015

Somayya Madakam, R. Ramaswamy, Siddharth Tripathi, Internet of Things (IoT): A Literature Review, Journal of Computer and Communications, 2015, 3, 164-173

Jacob Morgan ,A Simple Explanation Of 'The Internet Of Things', 2018, Forbes Magazin

Cisco Visual Networking Index Predicts Near-Tripling of IP Traffic by 2020. http://www.aaleuropeeu/projects/we-care/

Rodrigo Arias, Knud Lasse Lueth, Abhay Rastogi, The effect of the Internet of Things on sustainability, 2018

Maciej Kranz,6 ways the Internet of Things is improving our lives, january 2018 